\title{
Introduction to the Diffusion, Impacts, Adoption and Usage of ICTs upon Society Mini-track
}

\author{
Prof. Jyoti Choudrie \\ Systems Management Research \\ Unit (SyMRU) \\ Business School \\ University of Hertfordshire \\ UK \\ AL10 9EU \\ e-mail address: \\ j.choudrie@herts.ac.uk
}

\author{
Dr. Sherah Kurnia \\ Department of Computing \\ and Information Systems \\ University of Melbourne \\ Australia \\ e-mail address: \\ sherahk@unimelb.edu.au
}

\author{
Dr. Shahper Vodanovich \\ School of Computer and \\ Mathematical Sciences \\ Auckland University of \\ Technology \\ New Zealand \\ e-mail address: \\ svodanov@aut.ac.nz
}

Around the globe, Information and Communication Technologies (ICT) continue to expand rapidly and transform organizations, governments and individuals. Digital transformations experienced to date, have important implications for research and practice.

This mini track aims to draw researchers for sharing their research findings related to how ICTs are used, adopted and diffused within the society and their impacts on individuals, organizations and wider social communities around the world. The mini track helps academics, industry and government agencies to gain novel insights of how ICTs are currently being used, issues related to adoption and diffusion, ways of mitigation, as well as the consequences of ICT adoption within society.

Due to the importance of ICT use, adoption, diffusion and impacts, this mini track has constantly attracted researchers working in this area, with this being no exception. This year, there are four high quality papers that have been accepted

The first paper, Inclusive Innovation in the Private Sector: The Case of East African Tech Start-Ups, investigates in East Africa, the role of inclusive innovation in technology start-ups and shows the important role of contextual factors in driving start-ups to adopt methods supported by inclusive innovation.

The second paper, The Influence of Situation-Dependent Factors on Mobile Shopping Usage, examines a number of situational factors affecting the intention to use a mobile device for actual purchase transactions. Within the context of mobile devices, actual purchases appear to be the least adapted shopping activity.

The third paper, A Meta-Analysis of Enjoyment Effect on Technology Acceptance: The Moderating Role of Technology Conventionality, investigates the influence of perceived enjoyment on behavioural intention varying across conventional and unconventional/ innovative technology use. Findings showthat perceived enjoyment has a substantial role in shaping the behavioural intention of users for both conventional and unconventional technologies.

The final paper, Geographic Patterns and Socio-Economic Influences on Mobile Internet Access and Use in United States Counties, explores the use of mobile technologies and mobile internet for eentertainment and e-commerce purposes in the United States. The study identifies spatial dimensions of county-level mobile internet activity and the importance of market forces on mobile internet usage. 